\title{
Comparison of the Characteristics of Solid Type and Annular Type Nuclear Fuels Using Thermoelastic-Plastic-Creep FEM
}

\author{
Young-Doo Kwon, ${ }^{1}$ Dae-Suep Lee, ${ }^{2}$ and Tae-Hyeok Yun $^{3}$ \\ ${ }^{1}$ School of Mechanical Engineering \& IEDT, Kyungpook National University, 80 Daehakro, Bukgu, Daegu 702-701, Republic of Korea \\ ${ }^{2}$ School of Computer Aided Mechanical Engineering, Yeungjin College, Bukgu, Daegu 702-721, Republic of Korea \\ ${ }^{3}$ Computer Aided Mechanical Design, Gumi University, Gumi, Gyeongsangbuk-Do 730-711, Republic of Korea
}

Correspondence should be addressed to Dae-Suep Lee; dslee@yjc.ac.kr

Received 19 February 2016; Accepted 17 April 2016

Academic Editor: Zhiqiang Hu

Copyright (C) 2016 Young-Doo Kwon et al. This is an open access article distributed under the Creative Commons Attribution License, which permits unrestricted use, distribution, and reproduction in any medium, provided the original work is properly cited.

\begin{abstract}
The purpose of this study is to compare the characteristics of two types of nuclear fuel using the finite element program of thermoelastic-plastic-creep analysis. The analyzed fuel rods are of two types, solid and annular ones, and their thermomechanical characteristics are compared. Thermoelastic-plastic-creep analyses were made using an in-house finite element analysis program that adopts the "effective-stress-function" algorithm. The temperature-dependent material properties, which were obtained from the experiments for actual nuclear reactors, are adopted. The effects of type of fuel systems are revealed in both stresses and temperature distributions. The maximum tensile and compressive hoop stress of pellet and cladding are monitored to evaluate the mechanical behavior, and the maximum temperature is used to evaluate the thermal behavior. Although the annular type of fuel has certain disadvantage, it would be used very effectively or safely in future nuclear power plants.
\end{abstract}

\section{Introduction}

The recent nuclear fuels are of solid type $[1,2]$ and are generally utilized in the pressurized water nuclear reactors. Annular types of fuel [3-7] have been introduced to improve the performance of nuclear power plant. Annular fuels have wider surfaces, which promote the transfer of generated heat into the cooling water. Therefore, for the same overall heat generations, the surface temperature of the annular nuclear fuel can be reduced far more than conventional solid fuels, which means there is some extra margin to unexpected transient situations, such as Reactivity Initiated Accident (RIA) or Loss-of-Coolant Accident (LOCA) [8]. On the contrary, if both fuel types are allowed to similar fuel temperatures, the higher heat generation of the annular fuel may be possible to produce higher power than the solid type fuel.

Thermoelastic-plastic-creep analyses were conducted using an in-house thermoelastic-plastic-creep finite element program that adopted the "effective-stress-function (ESF)" algorithm [9], which appears to be one of the most powerful procedures for these tremendously complex thermomechanical phenomena. Two major iterations exist in the nonlinear plastic stress analysis [10]. One is equilibrium iteration; the other iteration tests the yield condition and examines the flow stress. Consequently, many coupled iteration cycles result in a large amount of computing time. The ESF algorithm does not adopt iterations to test yield and examine flow stress. Rather, it uses a simple bisectional algorithm to solve the effectivestress-function directly. Nonlinear iterative stress analyses were performed after nonlinear iterative temperature analyses in each time step. A semi-fully coupled algorithm was applied to this procedure, because the temperature distribution is rather insensitive to deformation compared to the stress distribution in general. Most analyses continued until after all the gaps disappeared.

In this study, thermoelastic-plastic-creep analyses of solid and annular type were performed under the same conditions, and the temperatures and stresses of the solid and annular pellets were compared. The pros and cons of the both types of fuel can be evaluated in terms of the temperatures and stresses of pellets. The overall characteristics of the annular fuels 
were evaluated by checking the thermomechanical behavior under given initial inner and outer gaps between the pellet and cladding. The narrowing inner and outer gaps between the pellet and cladding affected the temperature and stress distributions of the fuel system.

\section{Effective-Stress-Function Algorithm}

The governing equations of thermomechanical behavior are as shown in (1) and (2):

$$
\begin{array}{r}
{ }^{t+\Delta t} \mathbf{C}^{(i)}{ }^{t+\Delta t} \dot{\mathbf{T}}^{(i-1)}+\left({ }^{t} \mathbf{K}^{K}+{ }^{t} \mathbf{K}^{C}\right) \Delta \mathbf{T}^{(i)} \\
={ }^{t+\Delta t} \mathbf{Q}+{ }^{t+\Delta t} \mathbf{Q}^{C(i-1)}-{ }^{t+\Delta t} \mathbf{Q}^{K(i-1)},
\end{array}
$$

where $i$ is iteration number, ${ }^{t+\Delta t} \mathrm{C}$ is heat capacity matrix at time $t+\Delta t,{ }^{t+\Delta t} \mathbf{\mathbf { T }}$ is rate of temperature at time $t+\Delta t,{ }^{t} \mathbf{K}^{K}$ is conductivity matrix at time $t,{ }^{t} \mathbf{K}^{C}$ is convection matrix at time $t, \Delta \mathbf{T}$ is incremental nodal temperature, ${ }^{t+\Delta t} \mathbf{Q}$ is heat flux at time $t+\Delta t,{ }^{t+\Delta t} \mathbf{Q}^{C}$ is equivalent heat flux (convection) at time $t+\Delta t$, and ${ }^{t+\Delta t} \mathbf{Q}^{K}$ is equivalent heat flux (conduction) at time $t+\Delta t$. Consider the following:

$$
\begin{aligned}
& { }_{0}^{t+\Delta t} \mathbf{K}^{(i-1)} \Delta \mathbf{U}^{(i)} \\
& ={ }^{t+\Delta t} \mathbf{P}-{ }^{t+\Delta t} \mathbf{F}^{(i-1)} \\
& \quad-\mathbf{M}\left\{\frac{4}{(\Delta t)^{2}}\left({ }^{t+\Delta t} \mathbf{U}^{(i-1)}-{ }^{t} \mathbf{U}\right)-\frac{4}{\Delta t}{ }^{t} \dot{\mathbf{U}}-{ }^{t} \ddot{\mathbf{U}}\right\},
\end{aligned}
$$

where $\mathbf{M}$ is mass matrix, $\Delta \mathbf{U}$ is increment of the nodal displacement vector, ${ }^{t} \dot{\mathbf{U}}{ }^{t} \ddot{\mathbf{U}}$ is velocity and acceleration vector at time $t,{ }^{t+\Delta t} \mathbf{K}$ is stiffness matrix at time $t+\Delta t,{ }^{t+\Delta t} \mathbf{P}$ is external force vector at time $t+\Delta t$, and ${ }^{t+\Delta t} \mathbf{F}$ is incremental nodal force vector at time $t+\Delta t$.

The developed finite element module should be able to conduct thermoelastic-plastic-creep analyses because the pellet and cladding during normal or abnormal operation may exhibit plasticity and creep phenomena at high temperatures. To resolve the complex thermoelastic-plasticcreep finite element problem, the ESF algorithm [11] was adopted, as a stable, accurate, and computationally efficient procedure, which avoids the tedious coupled iterations and slow convergence due to the unknown flow stress. The von Mises flow stress in yield state is not obtained by iteration but obtained directly by solving nonlinear equation. Therefore, we can eliminate coupled iterations of equilibrium and flow stress. The main part of ESF algorithm of the Kojic and Bathe [11] is introduced here with slightly modified solution procedures but with the same nomenclatures. By including thermoelastic-plastic and creep deformations, the constitutive equations can be expressed as follows:

$$
\begin{aligned}
{ }^{t+\Delta t} \mathbf{S} & =\frac{{ }^{t+\Delta t} E}{1+{ }^{t+\Delta t} \nu}\left({ }^{t+\Delta t} \mathbf{e}^{\prime}-{ }^{t+\Delta t} \mathbf{e}^{P}-{ }^{t+\Delta t} \mathbf{e}^{C}\right), \\
{ }^{t+\Delta t} \sigma_{m} & =\frac{{ }^{t+\Delta t} E}{1-2^{t+\Delta t} \nu}\left({ }^{t+\Delta t} e_{m}-{ }^{t+\Delta t} e^{\text {th }}\right),
\end{aligned}
$$

where for time $t+\Delta t,{ }^{t+\Delta t} \mathbf{S}$ is deviatoric stress tensor, ${ }^{t+\Delta t} \mathbf{e}^{\prime}$ is deviatoric strain tensor, ${ }^{t+\Delta t} \mathbf{e}^{P}$ is plastic strain tensor, ${ }^{t+\Delta t} \mathbf{e}^{C}$ is creep strain tensor, ${ }^{t+\Delta t} \sigma_{m}$ is mean stress, ${ }^{t+\Delta t} e_{m}$ is mean strain, ${ }^{t+\Delta t} E,{ }^{t+\Delta t} v$ are Young's modulus and Poisson's ratio, and ${ }^{t+\Delta t} e^{\text {th }}$ is thermal strain.

Because the creep and plastic mean strains are zero, the mean stress is determined using (4), which does not involve inelastic strain.

The task of integrating the stress increments is reduced to designing an efficient method for determining ${ }^{t+\Delta t} \mathbf{S}, \Delta \mathbf{e}^{P}$, and $\Delta \mathbf{e}^{C}$. Using the $\alpha$-method $(\tau=t+\alpha \Delta t)$ with $\alpha=1$, the following can be derived:

$$
\Delta \mathbf{e}^{C}=\Delta t^{\tau} \gamma^{\tau} \mathbf{S}
$$

When the creep formula to be used is determined, the scalar function $\gamma^{\tau}$ can be calculated. In the case of isotropic hardening, the von Mises yield condition is represented by the following equation:

$$
{ }^{t+\Delta t} f_{y}\left({ }^{t+\Delta t} \sigma_{y}\right)=\frac{1}{2}{ }^{t+\Delta t} \mathbf{S}^{t+\Delta t} \mathbf{S}-\frac{1}{3}\left({ }^{t+\Delta t} \sigma_{y}\right)^{2}=0 .
$$

Next, let us consider the determination of the increase in plastic strain, $\Delta \mathbf{e}^{P}$. The flow rule of associative plasticity can be used in the following form:

$$
\Delta \mathbf{e}^{P}=\Delta \lambda^{t+\Delta t} \mathbf{S}
$$

and the nonlinear equation for the yield condition can be obtained in the following form:

$$
f\left({ }^{t+\Delta t} \bar{\sigma}\right)=a^{2 t+\Delta t} \bar{\sigma}^{2}+b^{\tau} \gamma-c^{2 \tau} \gamma^{2}-d^{2}=0 .
$$

The coefficients $b, c$, and $d$ are constants that depend only on the known values, which are independent of ${ }^{t+\Delta t} \bar{\sigma}$, whereas the value of the coefficient " $a$ " varies with ${ }^{t+\Delta t} \bar{\sigma}$ [11]. The function $f\left({ }^{t+\Delta t} \bar{\sigma}\right)$ defined by (8) is the effective-stressfunction, whose zero provides the solution for the flow stress ${ }^{t+\Delta t} \bar{\sigma}$. In particular, with this solution, the assumptions used to calculate the creep and plastic strain increments, (5) and (7), as well as the yield condition, (6), are satisfied.

A simple and stable bisection procedure acceleration scheme was used to solve the nonlinear equation (8). When ${ }^{t+\Delta t} \mathbf{S}, \Delta \mathbf{e}^{P}$, and $\Delta \mathbf{e}^{C}$ are obtained, the flow stress ${ }^{t+\Delta t} \bar{\sigma}$ is evaluated simultaneously. The determination steps of the flow stress, and so forth, using the ESF algorithm were modified from the original one of the Kojić and Bathe [11], for the faster convergence, and can be summarized as follows.

Solution steps in the effective-stress-function algorithm for thermoplasticity and creep [2] are as follows:

(1) Initialize value ${ }^{t+\Delta t} \bar{\sigma}^{(k=1)}={ }^{t} \sigma_{y}$ and then for $k=$ $2,3, \ldots$

(2) Compute ${ }^{\tau} \gamma^{(k)}$ from the creep formula. 


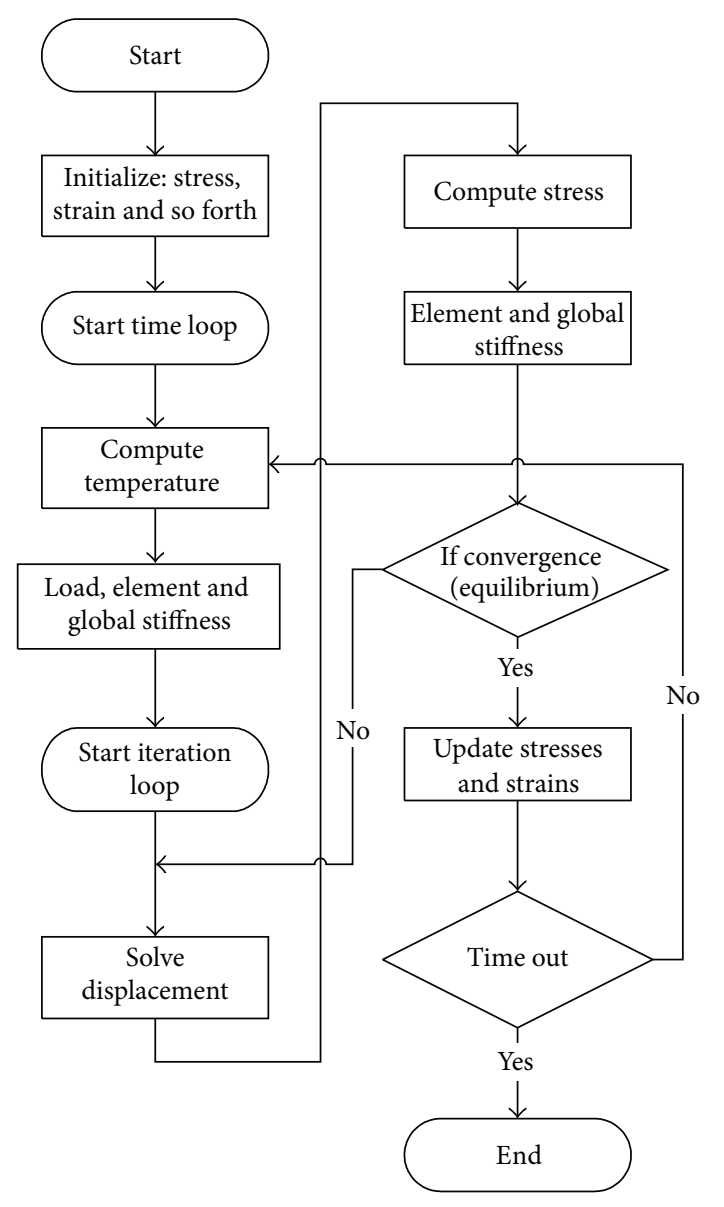

FIGURE 1: Flowchart of the ESF module.

(3) Compute $\Delta \lambda^{(k)}$ from the yield curve (when ${ }^{t+\Delta t} \bar{\sigma}^{(k)}>$ $\left.{ }^{t} \bar{\sigma}_{y}\right)$

calculate the value of the effective-stress-function $f\left({ }^{t+\Delta t} \bar{\sigma}^{(k)}\right)$,

compute $^{t+\Delta t} \bar{\sigma}^{(k)}$; here, one step of a bisection algorithm is used; if ${ }^{t+\Delta t} \bar{\sigma}^{(k)}$ does not represent (to a specified tolerance) the solution, go to (2),

step (3) should be sought and iterated at the same time.

(4) Compute ${ }^{t+\Delta t} \bar{\sigma},{ }^{t+\Delta t} \mathbf{S}, \Delta \mathbf{e}^{P}$, and $\Delta \mathbf{e}^{C}$.

2.1. Implementation of the Effective-Stress-Function. A coupled thermoelastic-plastic-creep analysis module was established using the Visual FORTRAN 6.0 language to implement the ESF algorithm in the FE solver, as shown in Figure 1. This module can conduct transient FE thermal analysis and nonlinear mechanical analysis, such as plastic and creep. To solve a global stiffness matrix effectively, the skyline method [10], which is suitable for a relatively small-size matrix, was employed.

In the module, there are two major loops used to carry out nonlinear thermal and structural analysis. The outer loop was used to progress the time steps. The inner loop was used to

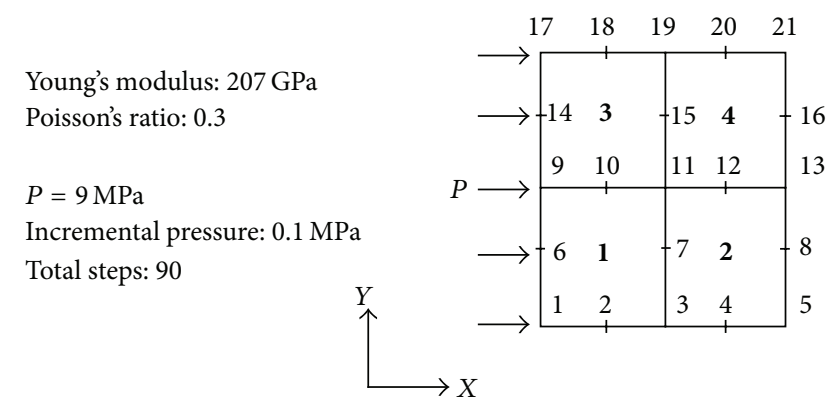

FIGURE 2: Axisymmetric FEM mesh and applied standard internal loads.

search for the nodal equilibrium state with updated stresses. When the stresses and strains during nonlinear behavior, such as plastic and creep, were updated, the ESF algorithm could be called to determine the effective stress for the current time step. A well-known simple bisectional algorithm was used to resolve the effective-stress-function. In addition, not a full but a quasi-fully coupled thermal-mechanical algorithm was applied to this procedure because the temperature distribution is rather insensitive to deformation compared to the stresses. The precision of the developed algorithm was compared with the commercial package ADINA for several simple test cases.

2.2. Verification of Present Program for Thermoelastic-PlasticCreep Analysis. Thermoelastic-plastic-creep analyses of the simple FEM models were carried out using the developed FORTRAN program and compared with the commercial program ADINA to verify the performance of overall procedures.

2.2.1. Plasticity. The developed FORTRAN program was tested with three types of materials: elastic perfect, bilinear, and general plasticity. Three cases of the mechanical analyses were made for the axisymmetric model under the same boundary conditions. As shown in Figure 2, the initial value of the yield stress is shown, and the mechanical load increases up to 90 steps $(9 \mathrm{MPa})$ of load with each increment of internal pressure of $0.1 \mathrm{MPa}$.

The stresses (von Mises, radial stress, and hoop stress) of developed present program and ADINA are compared in Figure 3, where the positional number in $X$-axis denote the Gauss points along the axis. In other words, number 2 means the mid sampling point of Gauss in element 1 . As shown in Figure 3, one can see uniaxial material stress first and then radial distribution of each stress. The material has the same initial yield stress of $25 \mathrm{MPa}$. For general plasticity, we treated it as general diagram in different way to the actual bilinear case. Since the applied load exceeds the yield point, the inner region of the cylinder is in plastic state denoted by the notation "P," and the outer elastic region is denoted by "E." We can find the coincidence of stress components and von Mises stresses obtained by the two compared finite element programs. Consequently, the suggested modified solution steps of the present program are proven to have a verified accuracy for the plasticity problem. 


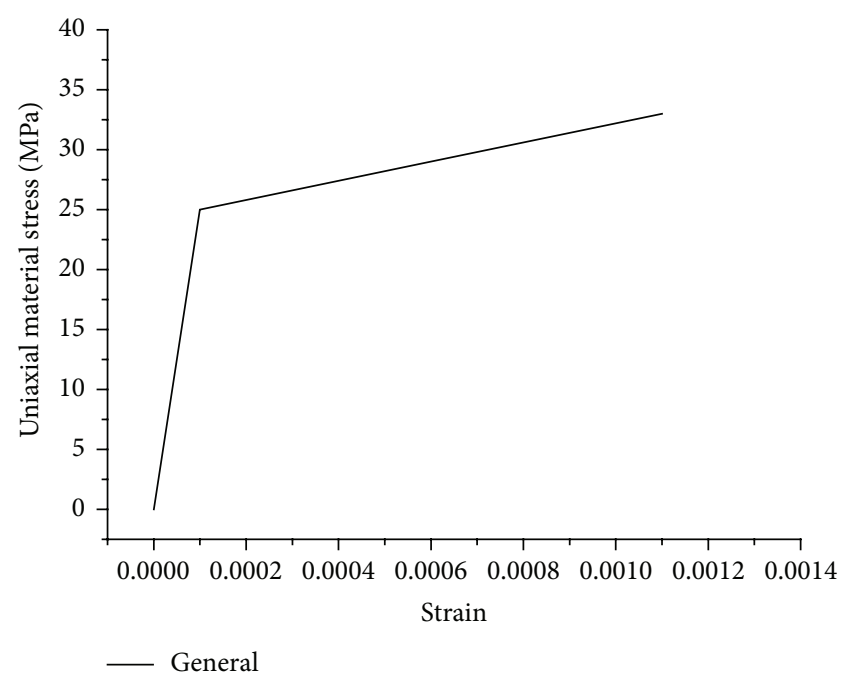

(a)
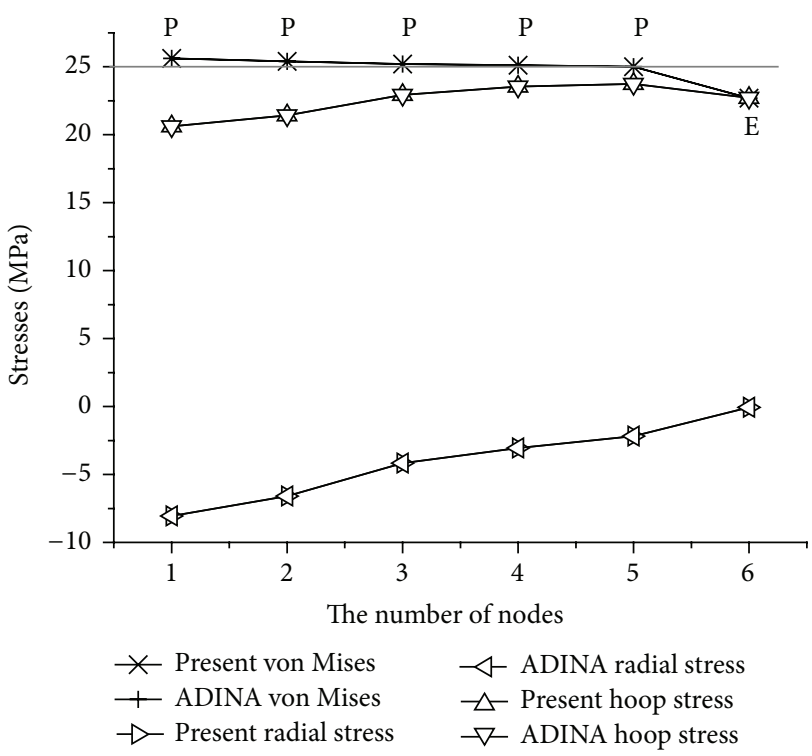

(b)

FIGURE 3: Comparison of stresses of present program and ADINA for general plasticity.



FIgURE 4: Boundary conditions and heat generation used in creep analysis.

2.2.2. Creep. To test the accuracy of the present program for creep problems without plasticity, the following power creep law was adopted, as shown in

$$
\bar{e}^{c}=a_{0} \bar{\sigma}^{a 1} t^{a 2}
$$

The creep analyses were performed both using present program and ADINA. The same boundary conditions (Figure 4) were applied in the creep analyses. The stresses (von Mises, radial stress, and hoop stress) of developed present program and ADINA are compared with each other for creep problems in Figure 5.

The positional numbers in the $X$-axis are the same as in Figure 3. The equivalent resulting stresses between the present program and ADINA mean that the developed program is reliable in the analysis of elastocreep as well as in the plasticity and thermal analyses.

\section{Modeling and Boundary Conditions of Solid and Annular Fuels}

The nuclear fuel rod consists of a pellet and cladding, and generates usable energy by causing the nuclear pellet to undergo a series of fission processes. During the power generation process, a thermal load caused by high temperature and a coolant load under high pressures affect the fuel rod, and the gap existing between pellet and cladding is reduced and disappeared to exhibit a complicated thermomechanical phenomena. To analyze these phenomena, the solid and annular nuclear fuel rods were modeled with pellet, claddings, and 


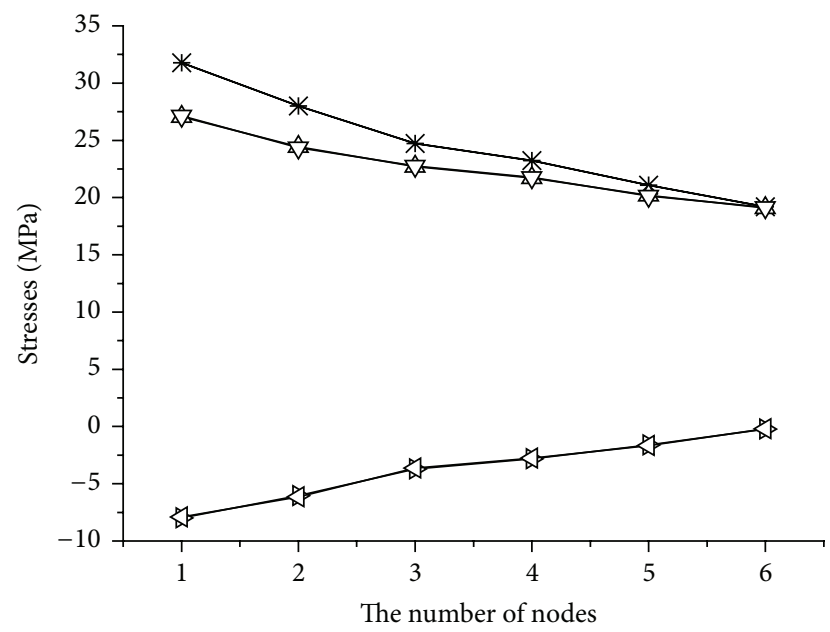

$$
\begin{array}{ll}
\leftarrow \text { Present von Mises } & \checkmark \text { ADINA radial stress } \\
\neg \text { ADINA von Mises } & - \text { Present hoop stress } \\
\rightarrow \text { Present radial stress } & -\checkmark \text { ADINA hoop stress }
\end{array}
$$

Figure 5: Comparison of stresses from present program and ADINA for elastocreep problem.

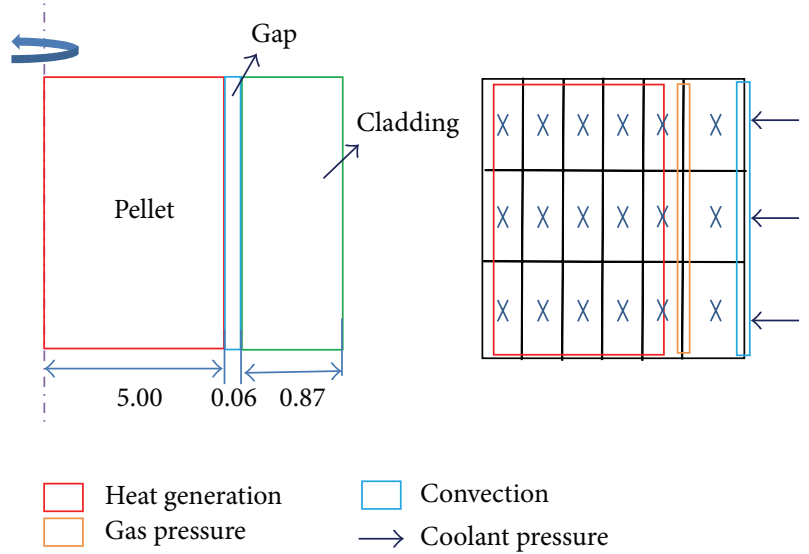

FIGURE 6: Solid nuclear fuel model and boundary condition [2].

gaps, as shown in Figures 6 and 7. Each section is discretized into several axisymmetric elements.

Mesh generation of the fuel rod was conducted using the quadratic 8-node elements, and the boundary conditions were applied consistently to them. Thermal boundary conditions comprise heat generation occurring from nuclear fission in the pellet and convection resulting from the coolant to the cladding. Mechanical boundary conditions are gas pressure in the gap and coolant pressures listed in Table 1.

\section{Thermal and Mechanical Material Properties}

The pellet and cladding that compose the fuel rod display complicated behavior due to heat expansion under high temperature and contraction under high coolant pressure. The physical properties applied to this analysis are adopted from

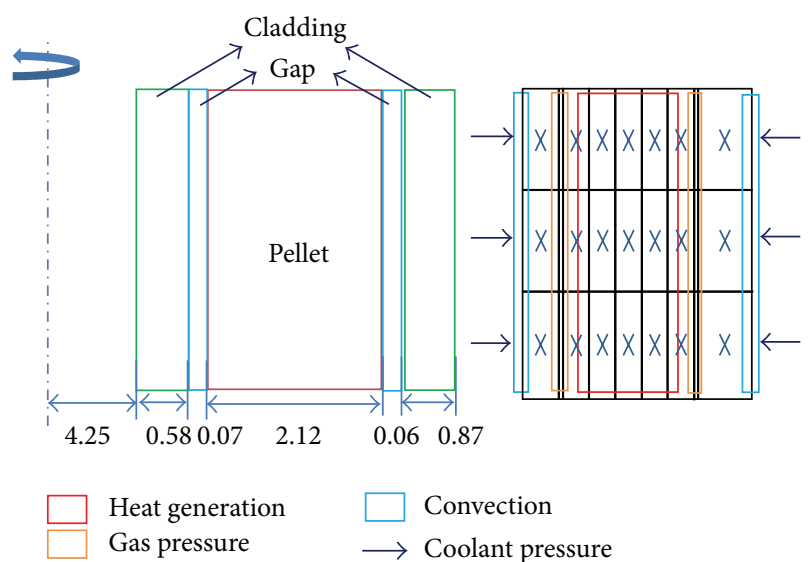

FIgURE 7: Annular nuclear fuel model and boundary condition [2].

TABLe 1: Applied pressures [2].

Coolant pressure

$15.5 \mathrm{MPa}$

Gap pressure

$3.45 \mathrm{MPa}$

TABLE 2: Mechanical properties at operating temperature.

\begin{tabular}{lcc}
\hline Mechanical & Cladding & Pellet \\
\hline Thermal expansion $\left[{ }^{\circ} \mathrm{K}^{-1}\right]$ & $0.672100 \times 10^{-5}$ & $0.910659 \times 10^{-5}$ \\
Young's modulus $[\mathrm{MPa}]$ & $0.795269 \times 10^{5}$ & $0.180316 \times 10^{6}$ \\
Poisson's ratio & 0.356756 & 0.316000 \\
Density $\left[\mathrm{Mg} \mathrm{mm}^{-3}\right]$ & $6.5500 \times 10^{-9}$ & $1.0412 \times 10^{-8}$ \\
\hline
\end{tabular}

[1]. Material properties of the pellet and cladding at operating temperature are given in Tables 2 and 3, and the heat transfer coefficients can be found in [4-7]. In the thermoelasticplastic-creep analysis, we used the mathematical formula as a function of temperature obtained from experiments in an actual nuclear reactor.

The cladding follows the creep law and creep properties of cladding are as follows:

$$
\begin{aligned}
& \dot{\varepsilon}=K \phi\left(\sigma+B e^{C \sigma}\right) \exp (-10,000 / R T) t^{-1 / 2} . \\
& \dot{\varepsilon} \text { : biaxial creep strain rate }\left[\mathrm{m} \mathrm{m}^{-1} \mathrm{~s}^{-1}\right] . \\
& K=5.129 \times 10^{-29}, B=7.252 \times 10^{2} . \\
& C=4.967 \times 10^{-8}, R=1.987\left[\mathrm{cal} \mathrm{mol}^{-1}{ }^{\circ} \mathrm{K}^{-1}\right] . \\
& T \text { : temperature }\left[{ }^{\circ} \mathrm{K}\right], t: \text { time }[\mathrm{s}] . \\
& \phi: \text { fast neutron flux }\left[\mathrm{n} \mathrm{m}^{-2} \mathrm{~s}^{-1}\right] . \\
& \sigma: \text { circumferential stress }[\mathrm{MPa}] .
\end{aligned}
$$

And it will yield above the yield stress and plasticity properties of cladding are as follows: 
TABLE 3: Thermal properties of sold/annular fuel.

\begin{tabular}{lccc}
\hline Thermal & Cladding & Gap & Pellet \\
\hline$T_{\infty}\left[{ }^{\circ} \mathrm{K}\right]$ & 562.7 & - & - \\
Heat generation $\left[\mathrm{mJ} \mathrm{mm}^{-3} \mathrm{~s}^{-1}\right]$ & - & - & 629.8 \\
Convection $\left[\mathrm{mJ} \mathrm{s}^{-1} \mathrm{~mm}^{-2}{ }^{\circ} \mathrm{K}^{-1}\right]$ & 100 & - & - \\
Specific heat $\left[\mathrm{mJ} \mathrm{Mg}^{-1}{ }^{-1} \mathrm{~K}^{-1}\right]$ & $0.325896 \times 10^{9}$ & $0.313889 \times 10^{9}$ & $0.313889 \times 10^{9}$ \\
Thermal conductivity $\left[\mathrm{mJ} \mathrm{s}^{-1} \mathrm{~mm}^{-1}{ }^{\circ} \mathrm{K}^{-1}\right]$ & $0.129064 \times 10^{2}$ & 0.344080 & $0.344808 \times 10$ \\
\hline
\end{tabular}

TABLE 4: Solid and annular types of fuel.

\begin{tabular}{lc}
\hline Solid type & Gap size $(0.06)$ \\
Annular type & Gap sizes $(0.07,0.06)$ \\
\hline
\end{tabular}

$$
\begin{aligned}
& \sigma_{y}=(37.135-0.0213 \times T) \times 9.81[\mathrm{MPa}] . \\
& \sigma=\sigma_{y}+(E / 10)\left(\varepsilon-\sigma_{y} / E\right)[\mathrm{MPa}] . \\
& \sigma_{y}: \text { yield stress }[\mathrm{MPa}] . \\
& \sigma \text { : true stress }[\mathrm{MPa}] \text { and } \varepsilon \text { : true strain. } \\
& E \text { : Young's modulus }[\mathrm{MPa}] . \\
& \left.T \text { : temperature }{ }^{\circ} \mathrm{K}\right] .
\end{aligned}
$$

\section{Finite Element Procedures for the Analyses of Nuclear Fuels}

Two types for the analyses were set up to perform thermalelastic-plastic-creep analysis as in Table 4 . The solid and annular type were analyzed for the standard gap sizes to see the thermomechanical behavior of the original annular fuel exactly.

The stresses of the pellet change with time because the gaps are reduced by contracting pellet creeping at high temperature or may be by yield. Thus, the sizes of the initial gaps in the annular fuel affect the maximum temperature of the pellet and also its maximum temperature. If we adjust them properly, we can obtain the result of smaller stresses and lower highest temperature of the pellet. This promising result may give us safer pellet against fracture and some extra margin to the thermal accident. When conducting thermoelasticplastic-creep analysis of solid and annular type, the gap sizes were given in Table 4.

5.1. Nonlinear Transient Thermal Analysis. Nonlinear transient thermal analysis with solid and annular type was conducted using the in-house FEM program adopting the transient thermal procedures [10]. As a result, the maximum temperature of solid nuclear fuels was $2,086^{\circ} \mathrm{K}$ and the maximum temperature of annular nuclear fuels was $835.5^{\circ} \mathrm{K}$. By applying the additional inner coolant in the annular nuclear fuels, overall heat convection was activated so much to cause a far less maximum temperature. Figures 8 and 9, respectively, show the temperature distribution of the solid and annular fuels from the center of the fuel to the outer cladding surface.

This implies that the annular type of fuel has a greater margin to an accidental situation against its failure. In

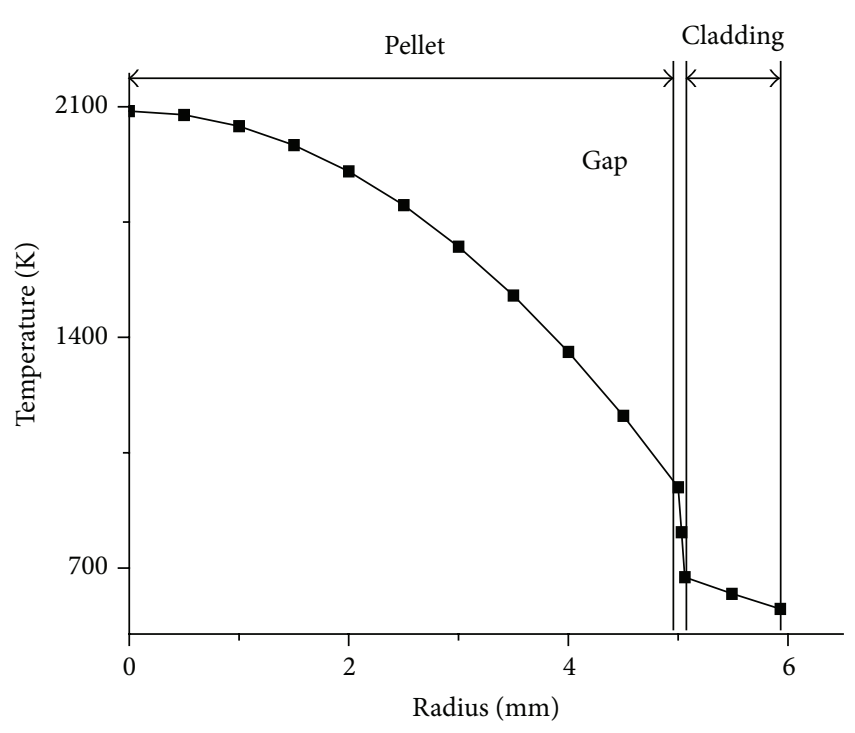

FIgURE 8: Temperature distribution of solid fuels.

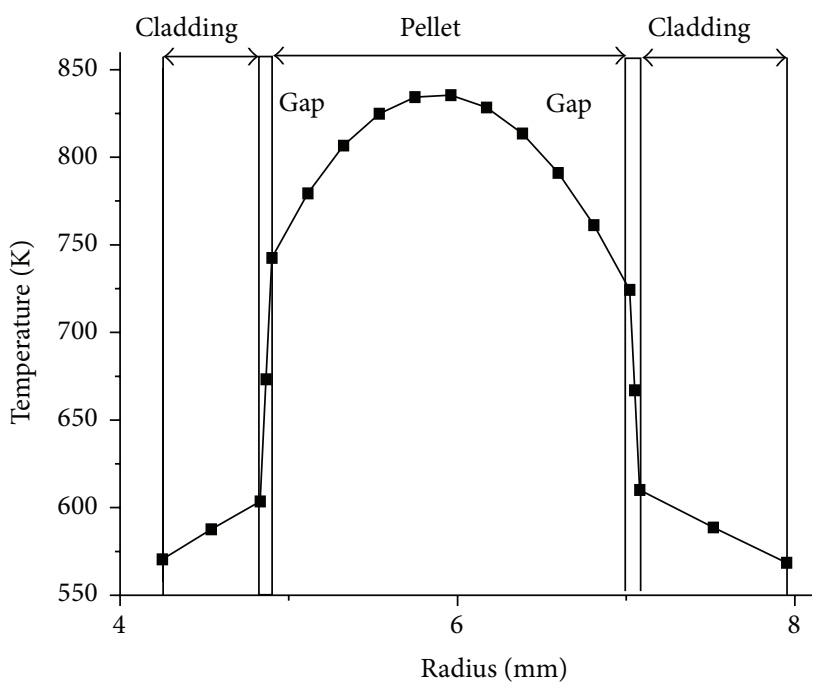

FIGURE 9: Temperature disribution of annular fuels.

the meantime, for the same margin of safety (i.e., similar maximum temperature of fuel), the annular fuel may be used with a higher heat generation to show higher power generation of the plant.

5.2. Nonlinear Thermoelastic-Plastic-Creep Analysis. Thermoelastic-plastic-creep analyses of the solid and annular fuels 

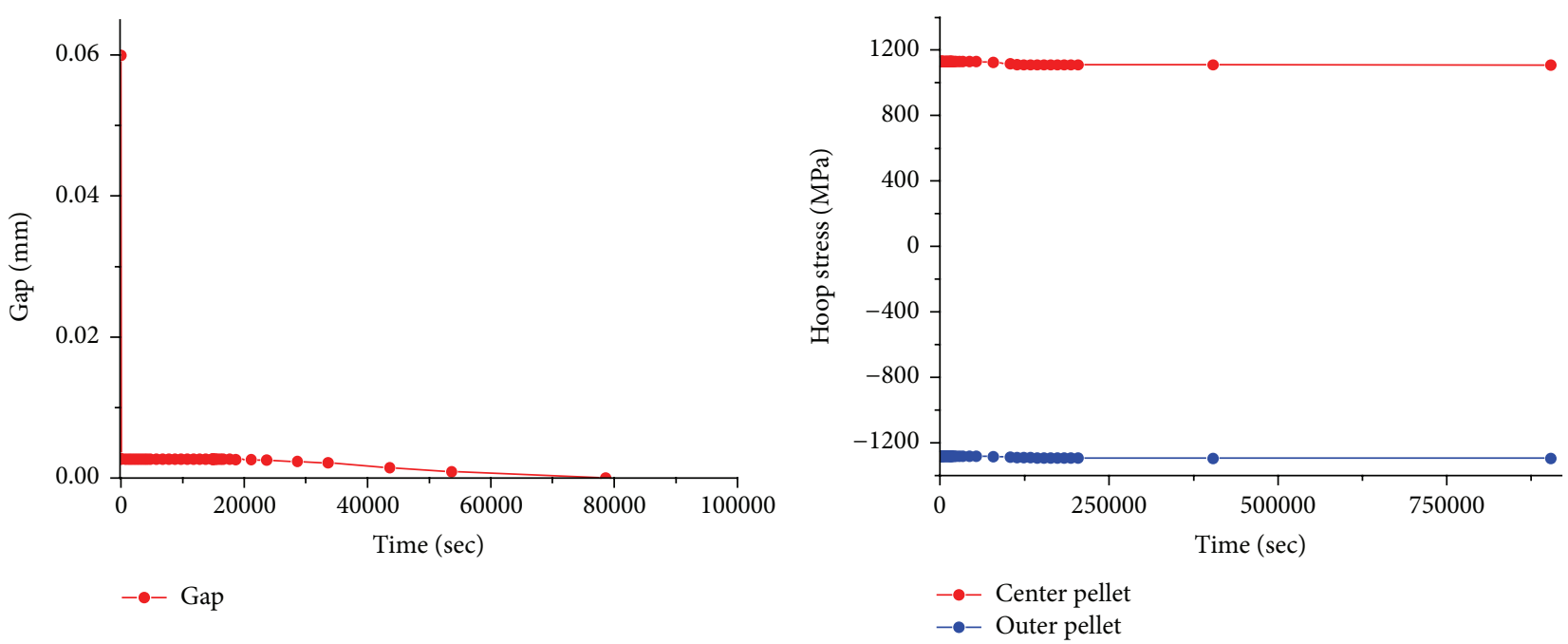

(a)
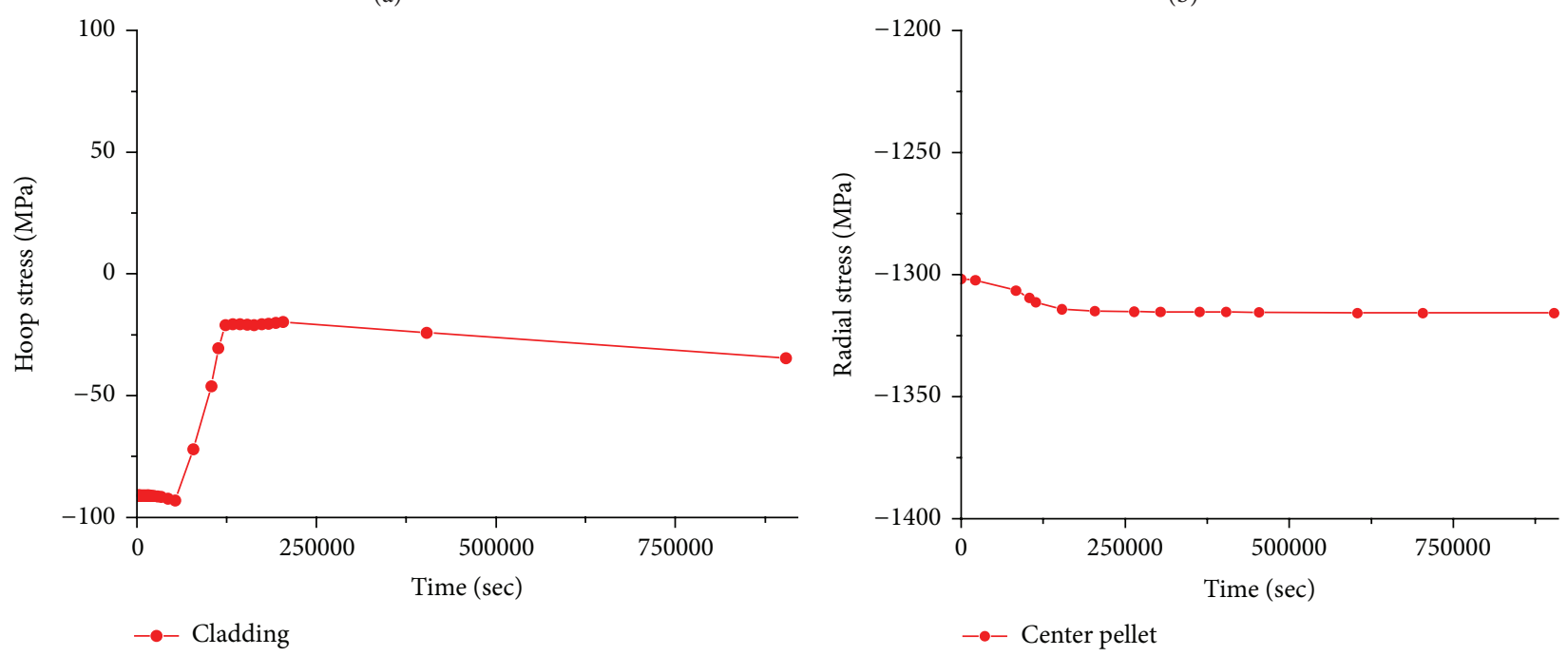

(c)

(d)

Figure 10: Analysis results of solid nuclear fuel ((a) gap size; (b) hoop stress of the pellet; (c) hoop stress of the cladding; (d) radial stress of the center-pellet).

were performed in connection with the thermal analysis. To see the exact thermomechanical behavior of annular fuel, the standard (temperature-dependent) method of analysis was adopted for the annular fuel of the standard gap sizes.

5.2.1. Analysis of the Solid Nuclear Fuel. The gap size between pellet and cladding of the solid nuclear fuel is $0.06 \mathrm{~mm}$. The maximum tensile hoop stress of the pellet was $1130.7 \mathrm{MPa}$ at the outer side. The maximum compressive hoop stress of the pellet was $1295.1 \mathrm{MPa}$ at the center of the pellet as in Figure 10. In the case of cladding, compressive stress occurred due to the coolant pressure. The maximum compressive hoop stress of the cladding was $93.8 \mathrm{MPa}$, and the hoop stress changed dramatically with the gap condition, which affect the temperature of the cladding. The gap between outer pellet and cladding was closed at 103,610 s.

5.2.2. Analysis of the Annular Nuclear Fuels. In thermoelastic-plastic-creep analysis, the maximum tensile hoop stress at the inner and outer pellet was 40.1 MPa and 94.7 $\mathrm{MPa}$, respectively, at initial stage due to thermal expansion as in Figure 11. The maximum compressive hoop stress at the inner pellet was $8.4 \mathrm{MPa}$ after the disappearance of the outer gap. In the cases of claddings, the inner one experiences the tensile stress and the outer one experiences compressive stress because of the coolant loads. The maximum tensile hoop stress of the inner cladding was $83.7 \mathrm{MPa}$, and this approaches to near zero after the contact in inner gap. The maximum compressive hoop stress of the outer cladding was $113.6 \mathrm{MPa}$, and this also approached to near zero after the contact at outer gap. The gap between outer pellet and cladding was closed on $283,610 \mathrm{~s}$, and the gap between inner pellet and cladding was closed on 703,610 s.

The mechanical behaviors of both types of fuel are listed in Table 5. The times of each gap closure are given, and also the maximum and minimum stresses of the pellet are given. The maximum pellet stress of the annular type of fuel is 94.7 MPa which is far smaller than 1130.7 MPa of the solid 


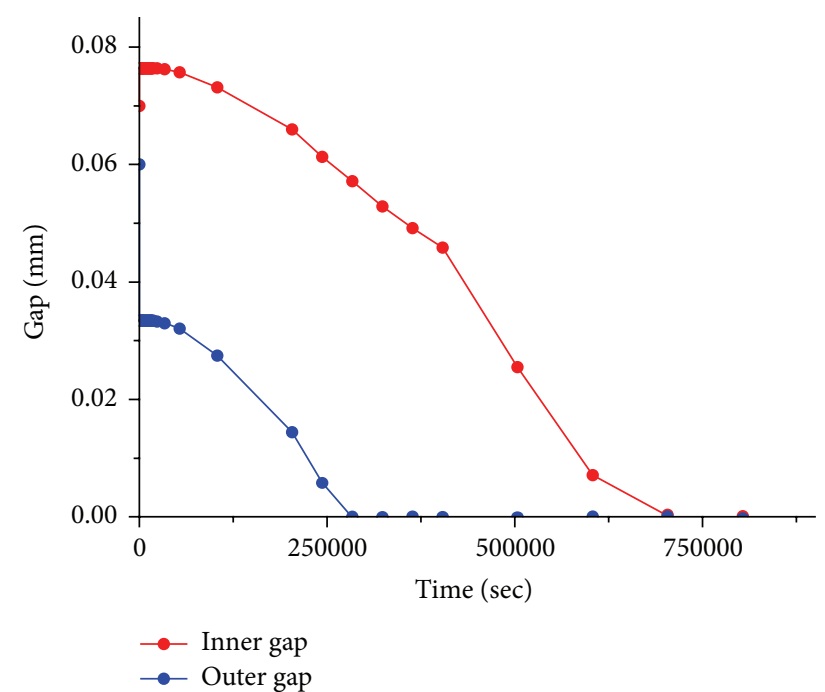

(a)

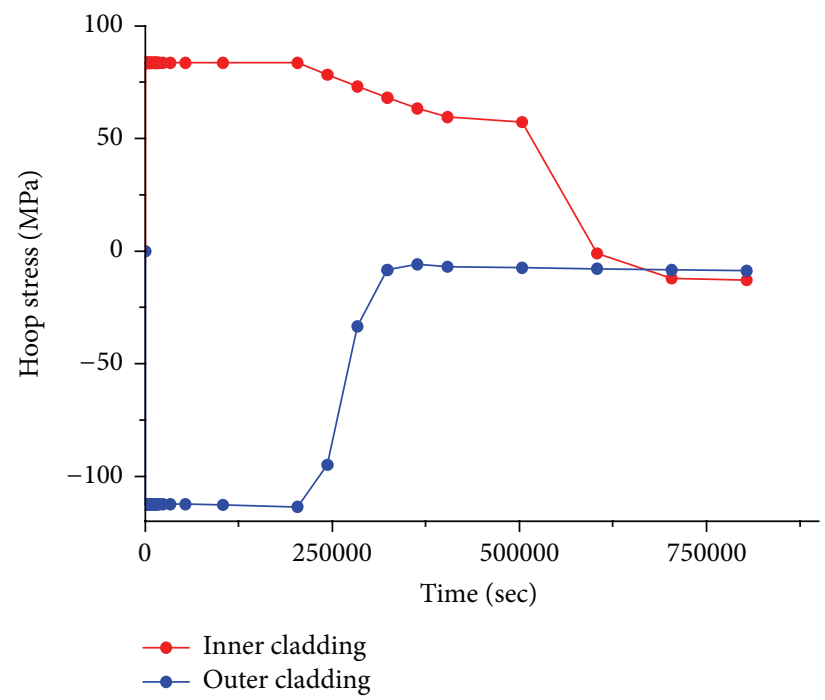

(c)

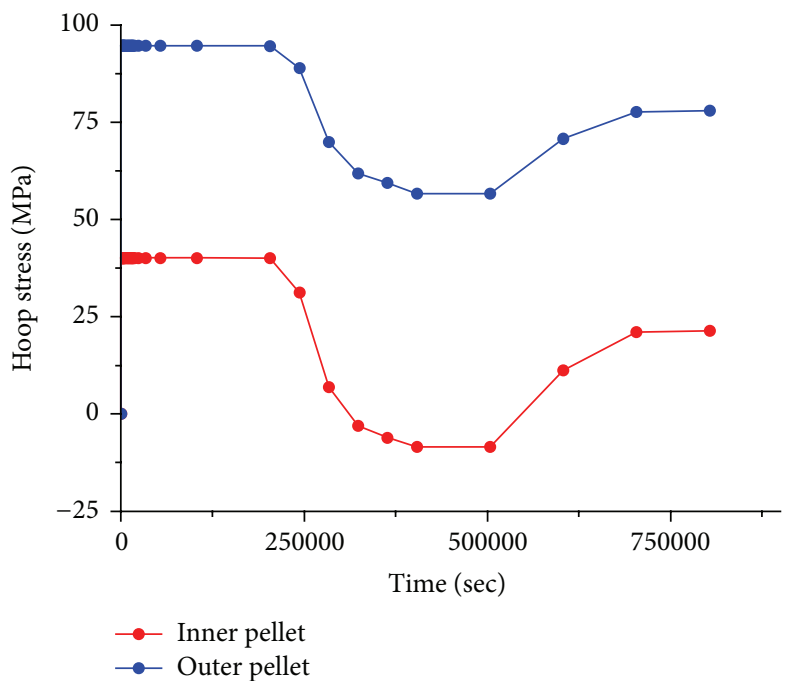

(b)

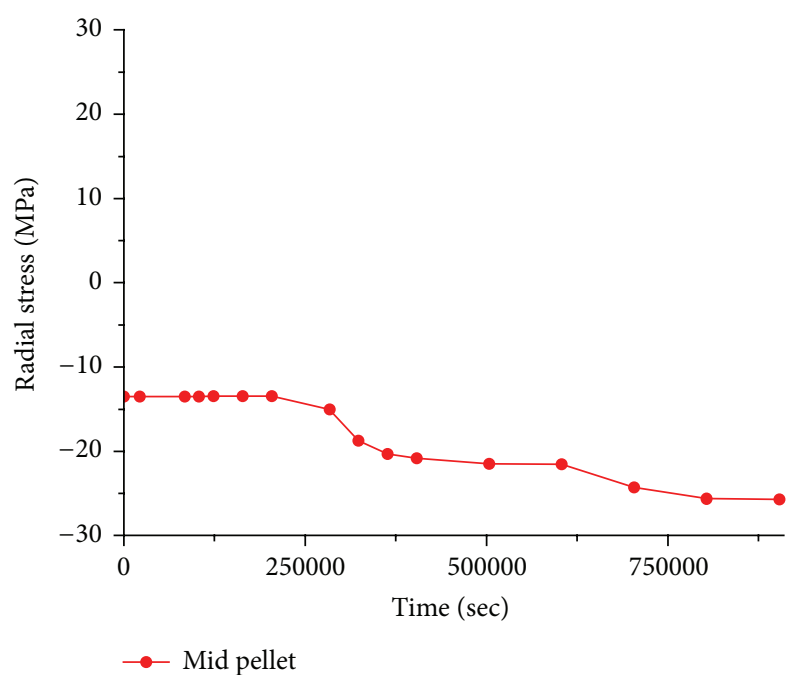

(d)

Figure 11: Analysis results of annular nuclear fuel ((a) gap size; (b) hoop stress of the pellet; (c) hoop stress of the cladding; (d) radial stress of the mid pellet).

TABLE 5: Comparison of the solid and annular nuclear fuels.

\begin{tabular}{cccc}
\hline & & Solid type & Annular type \\
\hline Gap contact time [step] & Inner gap & - & $703,610 \mathrm{~s}$ \\
& Outer gap & $103,610 \mathrm{~s}$ & $283,610 \mathrm{~s}$ \\
\hline Max. compressive stress of pellet & & $1295.1 \mathrm{MPa}$ & $8.4 \mathrm{MPa}$ \\
\hline Max. tensile stress of pellet & $1130.7 \mathrm{MPa}$ & $94.7 \mathrm{MPa}$ \\
\hline Max. absolute stress of cladding & & $93.8 \mathrm{MPa}$ & $113.6 \mathrm{MPa}$ \\
\hline Max. temperature & $2086.7^{\circ} \mathrm{K}$ & $835.5^{\circ} \mathrm{K}$ \\
\hline
\end{tabular}

type. This smaller maximum stress of the pellet dominantly reduces the chance of pellet fracture which might lead to the tearing of cladding. The absolute stress of the cladding of the annular fuel is also smaller than that of the solid fuel. Therefore, the mechanical characteristic of the annular fuel is very beneficial to be adopted in the nuclear power plant. This kind of favorable stress distribution is thought to be originated from the safe of the annular pellet, both cooling surfaces of cladding, and overall lower temperature distribution.

In the meantime, the maximum temperatures of the pellet in the solid and annular types of fuel are compared in Table 5. 
Because of the very wide convective surface of cladding, the annular type exhibits far less maximum temperature of $835.5^{\circ} \mathrm{K}$ than $2086.7^{\circ} \mathrm{K}$ of solid type. This will provide a maximum margin of safety in an accidental situation of the nuclear power plant.

In other words, suppose a LOCA happened in a plant. The critical time of melting in the case of annular fuel is far longer than that of the case of solid fuel. That difference of so called golden time is very important to the safety of the power plant and to a greater region of country, as we see in the Fukushima territory of the northeastern Japan.

If the same maximum temperature is applied to the annular type of fuel, it may adopt a higher overall heat generation per rod to result in higher efficiency of the nuclear power plant.

However, the annular type has a certain disadvantage too. It needs more space for stacking in the bundle of fuel rods, and a complicated fabrication technique would be required. If these technical difficulties are resolved anyway, it can serve as a promising type of nuclear fuel.

\section{Conclusion}

A finite element program adopting the ESF algorithm has been developed and compared with the commercial package ADINA to result in a perfect coincident for thermoelastoplastic-creep problems. We applied this FEA program to the analyses of conventional solid and annular types of nuclear fuel. The results are summarized as follows:

(1) The annular fuel exhibited far less maximum temperature of $835.5^{\circ} \mathrm{K}$ than $2086.7^{\circ} \mathrm{K}$ of solid type for the same overall heat generation. This characteristic will provide a generous margin to an accidental situation of nuclear power plant.

(2) The maximum hoop stress in the pellet of the annular type also was $94.7 \mathrm{MPa}$, which is far less than $1130.7 \mathrm{MPa}$ of the solid type. This will reduce the chance of pellet fracture which may result in the tear of cladding.

(3) The annular type of fuel needs more space than solid type and requires more or less complicate fabrication. However, it would be used very safely for the same overall heat generation or very effectively to produce higher power for the same level of safety.

\section{Competing Interests}

The authors declare that they have no competing interests.

\section{Acknowledgments}

This research was supported by Basic Science Research Program through the National Research Foundation of Korea (NRF) funded by the Ministry of Education (NRF2012R1A1A2008903). Authors are partially supported by BK21 plus project by the National Research Foundation of Korea.

\section{References}

[1] M. Suzuki and H. Saitou, Light water reactor fuel analysis code FEMAXI-IV (Ver. 2), JAERI, Data, Code, 1997.

[2] Y. D. Kwon, S. B. Kwon, H. J. Song, M. S. Kim, and J. S. Kim, Evaluation of Nuclear Fuel Behaviors Using Finite Element, Korea Atomic Energy Research Institute, Daejeon, Republic of Korea, 2010.

[3] Y. S. Yang, C. H. Shin, T. H. Chun, and K. W. Song, "Evaluation of a dual-cooled annular fuel heat split and temperature distribution," Journal of Nuclear Science and Technology, vol. 46, no. 8, pp. 836-845, 2009.

[4] Y. D. Kwon, Y. S. Yang, J. S. Kim, and S. B. Kwon, "A study on the flow characteristics in an annular type fuel pellet of PWR," Journal of Mechanical Science and Technology, vol. 27, no. 1, pp. 257-261, 2013.

[5] K. Takase, "Numerical prediction of augmented turbulent heat transfer in an annular fuel channel with repeated two-dimensional square ribs," Nuclear Engineering and Design, vol. 165, no. 1-2, pp. 225-237, 1996.

[6] Y.-D. Kwon, S.-B. Kwon, S.-S. Kim, and H.-J. Cho, "Optimization of gap sizes for the high performance of annular nuclear fuels," Journal of Mechanical Science and Technology, vol. 29, no. 4, pp. 1399-1405, 2015.

[7] C.-H. Shin, T.-H. Chun, D.-S. Oh, and W.-K. In, "Thermal hydraulic performance assessment of dual-cooled annular nuclear fuel for OPR-1000," Nuclear Engineering and Design, vol. 243, pp. 291-300, 2012.

[8] J. R. Lamarsh and A. J. Baratta, Introduction to Nuclear Engineering, Pearson, 2001.

[9] M. Kojić and K.-J. Bathe, “The 'effective-stress-function' algorithm for thermo-elasto-plasticity and creep," International Journal for Numerical Methods in Engineering, vol. 24, no. 8, pp. 1509-1532, 1987.

[10] K. J. Bathe, 'Finite Element Procedures, Prentice Hall, Englewood Cliffs, NJ, USA, 1996.

[11] M. Kojić and K. J. Bathe, Inelastic Analysis of Solids and Structures, Springer, Berlin, Germany, 2005. 


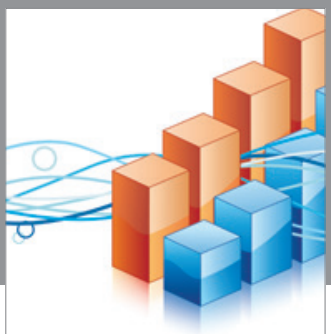

Advances in

Operations Research

vatem alat4

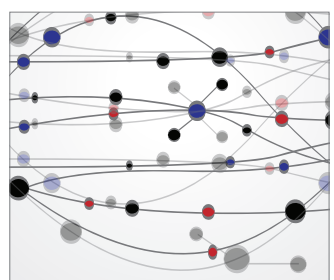

\section{The Scientific} World Journal
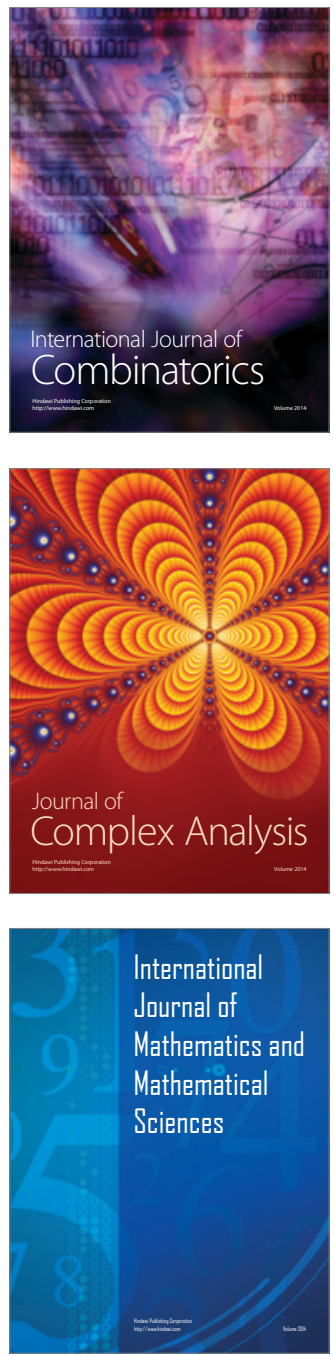
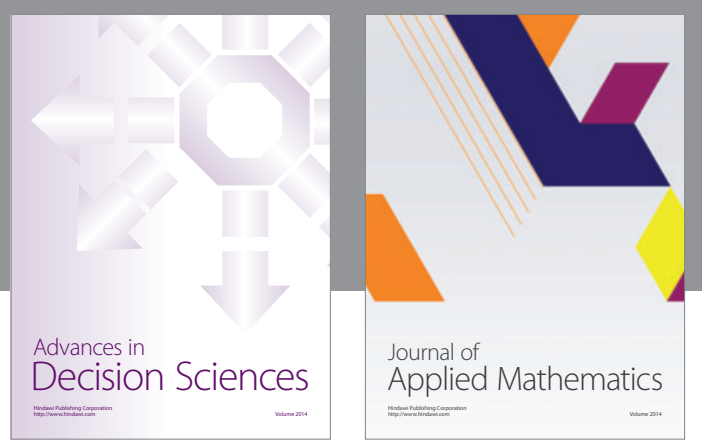

Algebra



\section{Hindawi}

Submit your manuscripts at

http://www.hindawi.com
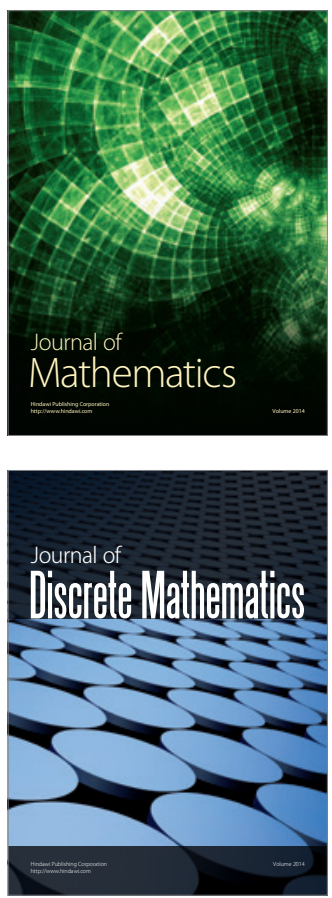

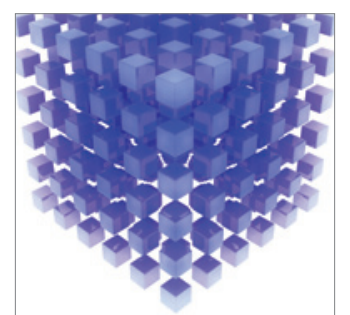

Mathematical Problems in Engineering
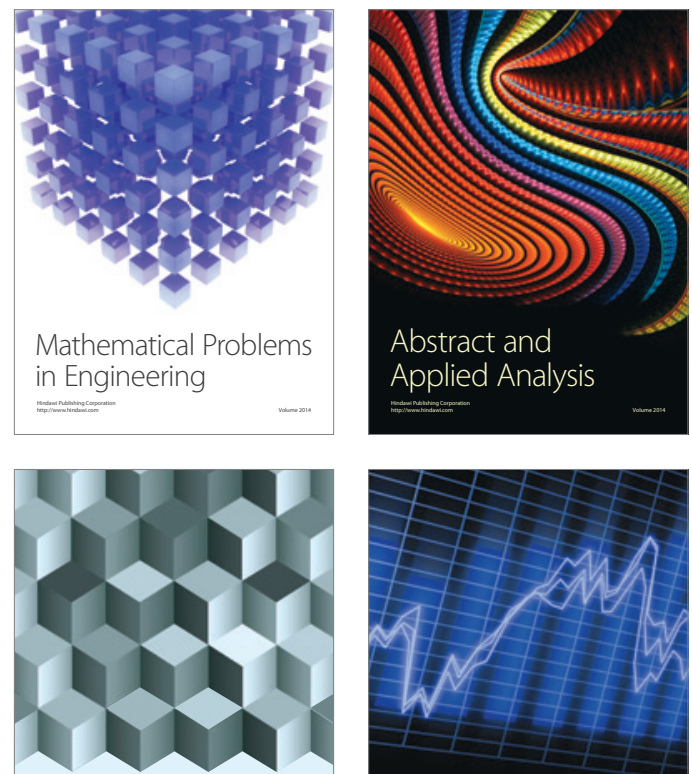

Journal of

Function Spaces

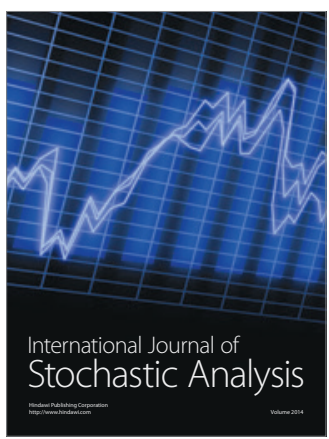

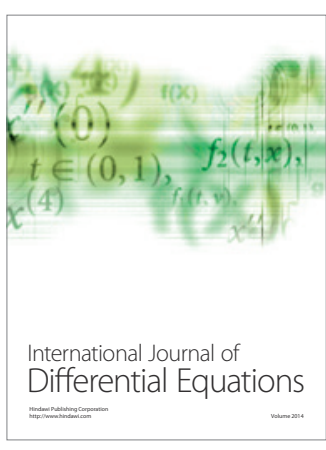
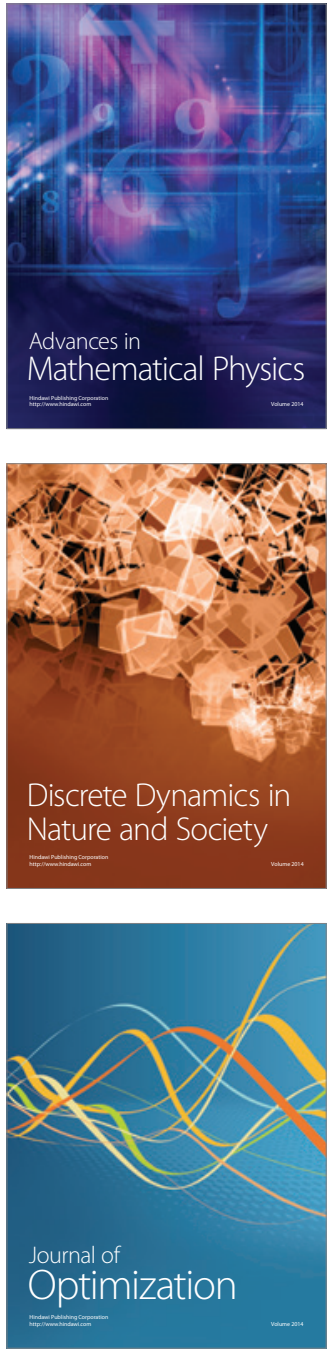